\title{
Coupled Tensor Model of Atrial Fibrillation ECG
}

\author{
Pedro Marinho R. de Oliveira and Vicente Zarzoso \\ Université Côte d'Azur, CNRS, I3S Laboratory \\ CS 40121, 06903 Sophia Antipolis Cedex, France \\ Email: $\{$ marinho, zarzoso $\}$ i3s.unice.fr
}

\author{
Carlos Alexandre R. Fernandes \\ Universidade Federal do Ceará \\ Sobral, Brazil \\ Email: alexandrefernandes@ufc.br
}

\begin{abstract}
Atrial fibrillation (AF) is the most frequent cardiac arrhythmia diagnosed in clinical practice, identified by an uncoordinated and irregular atrial depolarization. However, its electrophysiological mechanisms are still not clearly understood, increasing the intensive clinical research into this challenging cardiac condition in the past few years. The noninvasive extraction of the atrial activity (AA) from multi-lead electrocardiogram (ECG) recordings by signal processing techniques has helped in better understanding this complex arrhythmia. In particular, tensor decomposition techniques have proven to be powerful tools in this task, overcoming the limitations of matrix factorization methods. Exploring the spatial as well as the temporal diversity of ECG recordings, this contribution puts forward a novel noninvasive $A A$ extraction method that models consecutive AF ECG segments as a coupled block-term tensor decomposition, assuming that they share the same spatial signatures. Experiments on synthetic and real data, the latter acquired from persistent $A F$ patients, validate the proposed coupled tensor approach, which provides satisfactory performance with reduced computational cost.

Index Terms-Atrial Fibrillation, Blind Source Separation, Block Term Decomposition, Coupled Tensor Model, Electrocardiogram
\end{abstract}

\section{INTRODUCTION}

Representing a major health and social concern, atrial fibrillation $(\mathrm{AF})$ is a supraventricular tachyarrhythmia characterized by an uncordinated and irregular atrial activation [1]. With its electrophysiological mechanisms still not completely understood, the number of patients suffering from AF is increasing each year worldwide. In 2010, it was estimated that this number is around 33.5 million [2] and, if the number of hospitalizations per year stays stable, by $2050 \mathrm{AF}$ can become a new epidemic [3]. Considering this worrisome scenario, the intensive clinical research into this challenging cardiac rhythm disturbance has increased in the past decades and is expected to increase further in the following years.

In an AF electrocardiogram (ECG), the P wave that corresponds to a normal atrial activity (AA) during normal sinus rhythm is replaced by $\mathrm{f}$ waves (fibrillatory waves), which are present throughout the whole ECG recording. However, they are masked by the QRS-T complex, which represents the ventricular activity (VA), in each heartbeat. Precisely analyzing and characterizing the $\mathrm{f}$ waves is essential to better understanding the complex mechanisms behind AF.

Over recent years, signal processing techniques to noninvasively extract the AA from the standard 12-lead ECG have

Pedro Marinho R. de Oliveira is funded by a PhD scholarship from the IT Doctoral School (EDSTIC) of Université Côte d'Azur. helped cardiologists to better manage this cardiac condition. Since the AA extraction from multi-lead AF ECGs was shown to accept a blind source separation (BSS) formulation in [4], several methods based on matrix decompositions to solve BSS problems were reported in the literature as useful tools in noninvasive AA extraction for AF analysis [4], [5].

Overcoming the constraints imposed by matrix decompositions to guarantee their uniqueness, tensor decompositions can be essentially unique under more relaxed constraints. The block term decomposition (BTD) built from Hankel matrices has been recently proven to overcome the matrix-based techniques in the noninvasive extraction of the AA signal from synthetic and real AF ECGs [6], [7]. The Hankel-based BTD proposed as a technique to solve BSS problems in [8] suits the characteristics of AA in AF episodes, since atrial signals can be approximated by all-pole (or exponential) models and mapped onto Hankel matrices with rank equal to the number of poles [6].

Coupled tensor decompositions have been gaining space in biomedical signal processing problems [9], [10]. Aiming to further explore the spatial and temporal diversity of ECG signals, the present work puts forward the coupled BTD [11] as a novel noninvasive AA extraction method, modeling consecutive AF ECG segments, assuming that they share the same spatial signatures. To the best of the authors' knowledge, no coupled tensor approach has yet been applied to ECG signal processing. Also, this is the first time that the coupled BTD is used in a biomedical signal processing scenario.

Coupled BTD is assessed in synthetic signals that simulate an AF ECG and validated in real ECG recordings from two patients suffering from persistent AF, a complex case of this arrhythmia. In both scenarios, the coupled BTD is compared with the Hankel-based BTD (called hereafter BTD) regarding the AA extraction peformance and computational cost. Experimental results using Monte Carlo simulations evaluate the performance of both techniques, showing that the coupled approach provides an improved source separation with reduced computational cost.

The rest of this paper is organized as follows. Section II recalls the coupled BTD and the modeling of ECG data matrices as coupled tensors, while Section III assesses the coupled tensor approach using synthetic AF signals. Section IV presents quantitative measurements of AA content, the database, and the setup used in the experiments with real data, along with the results. Finally, Section V concludes this work 
and presents the prospects of future research.

\section{AF ECG AS A COUPLED BTD}

An ECG recording from $K$ leads composed by $M$ time samples can be viewed as a matrix:

$$
\mathbf{Y}=\mathbf{H S} \in \mathbb{R}^{K \times M}
$$

where $\mathbf{H} \in \mathbb{R}^{K \times R}$ is the mixing matrix, representing the spatial signature of the sources, $\mathbf{S} \in \mathbb{R}^{R \times M}$ is the source matrix that contains atrial, ventricular and other interference sources (noise, respiration, etc.), and $R$ is the number of sources. Since the goal is to estimate $\mathbf{H}$ and $\mathbf{S}$ from the matrix $\mathbf{Y}$, one can see that the signal estimation in ECG recordings can be considered as a BSS problem [4].

Proposed as a technique to solve BSS problems like (1), the BTD of an arbitrary third-order tensor $\mathcal{T} \in \mathbb{R}^{I_{1} \times I_{2} \times I_{3}}$ is given by [8]:

$$
\mathcal{T}=\sum_{r=1}^{R} \mathbf{E}_{r} \circ \mathbf{c}_{r}=\sum_{r=1}^{R}\left(\mathbf{A}_{r} \mathbf{B}_{r}^{T}\right) \circ \mathbf{c}_{r}
$$

where $\mathbf{c}_{r} \in \mathbb{R}^{I_{3}}$ is nonzero and $\mathbf{E}_{r} \in \mathbb{R}^{I_{1} \times I_{2}}$ has rank $L_{r}$, admitting the factorization $\mathbf{E}_{r}=\mathbf{A}_{r} \mathbf{B}_{r}^{T}$, where $\mathbf{A}_{r} \in \mathbb{R}^{I_{1} \times L_{r}}$ and $\mathbf{B}_{r} \in \mathbb{R}^{I_{2} \times L_{r}}$ are also rank- $L_{r}$ matrices. One can see that the BTD is a decomposition of $\mathcal{T}$ in multilinear rank- $\left(L_{r}, L_{r}, 1\right)$ terms, represented by a sum of the outer product of its matrix and vector factors.

For the particular case of AF ECGs, the third-order tensor $\mathcal{T}$ is the tensorization of the ECG data matrix $\mathbf{Y}$ by mapping each of its rows into a Hankel matrix and stacking them as frontal slices of $\mathcal{T}$. Matrices $\mathbf{E}_{r}$ have a Hankel structure, built from each source of $\mathbf{S}$, and vectors $\mathbf{c}_{r}$ represent each column of the mixing matrix $\mathbf{H}$. The Hankel structure of the source matrices suits the characteristics of AA in AF episodes, as atrial signals have an all-pole harmonic structure and, when mapped onto Hankel matrices, the number of poles matches the rank of the matrix [6].

Assuming that consecutive segments of an AF ECG recording have the same spatial signature, i.e., the same mixing matrix (which implies the same number of sources), they can be modeled as coupled BTD tensors, sharing the same nonzero vectors $\mathbf{c}_{r}$. Ideally, this assumption is valid for moderatelength ECG recordings of a single patient, given a fixed placement of the electrodes. Dividing an ECG recording in $N$ consecutive segments divides the original tensor in $N$ smaller tensors. For the case where the segments are of same length, the total number of scalar entries is reduced by a factor of $\approx 1 / N$. Jointly processing the $N$ third-order tensors is expected to reduce the computational cost, comparing as if they are processed separately. Also, the non-stationarity of AA signals during AF allows the exploitation of the temporal diversity, as the signals will be jointly estimated by the coupled tensor approach with different time signatures.

Considering that the matrix factors $\mathbf{A}_{r, n} \mathbf{B}_{r, n}^{T}$ have rank $L_{r, n}$ and $\mathbf{c}_{r}$ is a common factor, the coupled BTDs of $\mathcal{T}^{(n)} \in$

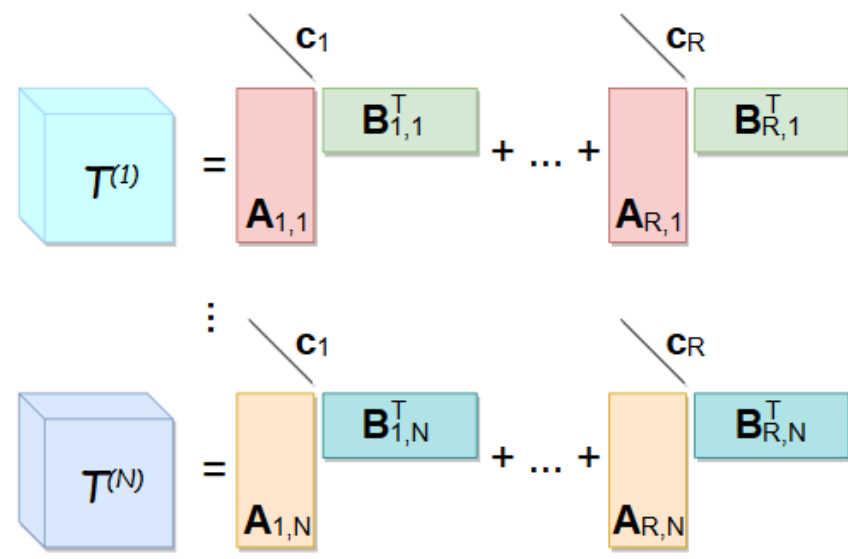

Fig. 1: Visual representation of the coupled BTD of arbitrary third-order tensors with common factor $\mathbf{c}_{r}$, for $r=1,2, \ldots, R$.

$\mathbb{R}^{I_{n} \times J_{n} \times K}$, for $n \in 1, \ldots, N$, can be written in the following form [11]:

$$
\mathcal{T}^{(n)}=\sum_{r=1}^{R}\left(\mathbf{A}_{r, n} \mathbf{B}_{r, n}^{T}\right) \circ \mathbf{c}_{r} .
$$

Some algebraic manipulations show that the overall coupled BTD accepts the matrix representation:

$$
\mathbf{T}=\left[\left(\mathbf{T}_{1}^{(1)}\right)^{T}, \ldots,\left(\mathbf{T}_{1}^{(N)}\right)^{T}\right]^{T}=\mathbf{F} \mathbf{C}^{T} \in \mathbb{R}^{\left(\sum_{n=1}^{N} I_{n} J_{n}\right) \times K}
$$

where $\mathbf{T}_{1}^{(n)}$ is the mode-1 matrix unfolding of $\mathcal{T}^{(n)}$, for $n=$ $1, \ldots, N, \mathbf{C}=\left[\mathbf{c}_{1}, \ldots, \mathbf{c}_{R}\right] \in \mathbb{R}^{K \times R}$, and $\mathbf{F} \in \mathbb{R}^{\left(\sum_{n=1}^{N} I_{n} J_{n}\right) \times R}$ is given by:

$$
\mathbf{F}=\left[\begin{array}{ccc}
\operatorname{vec}\left(\mathbf{B}_{1,1} \mathbf{A}_{1,1}^{T}\right) & \ldots & \operatorname{vec}\left(\mathbf{B}_{R, 1} \mathbf{A}_{R, 1}^{T}\right) \\
\vdots & \ddots & \vdots \\
\operatorname{vec}\left(\mathbf{B}_{1, N} \mathbf{A}_{1, N}^{T}\right) & \ldots & \operatorname{vec}\left(\mathbf{B}_{R, N} \mathbf{A}_{R, N}^{T}\right)
\end{array}\right] .
$$

The coupled BTDs in (3) are illustrated in Figure 1 and can be arbitrarily permuted. Uniqueness conditions for the overall coupled BTD tensor with $\mathbf{c}_{r}$ as a common factor are presented in [11]. For example, two necessary conditions for the uniqueness of the coupled BTD of (3) are:

1) $k_{\mathbf{C}} \geq 2$, where $k_{\mathbf{C}}$ is the Kruskal rank of the matrix $\mathbf{C}=\left[\mathbf{c}_{1}, \ldots, \mathbf{c}_{R}\right]$.

2) Matrix $\mathbf{F}$ has full column rank.

For the particular case of ECGs, we have $\mathbf{C}=\mathbf{H} \in \mathbb{R}^{K \times R}$, $\mathbf{A}_{r, n} \in \mathbb{R}^{I_{n} \times L_{r, n}}$ and $\mathbf{B}_{r, n} \in \mathbb{R}^{J_{n} \times L_{r, n}}$, where $I_{n}+J_{n}=$ $\left(M_{n}+1\right)$, with $\sum_{n=1}^{N} M_{n}=M$. Hence:

1) A necessary condition for $k_{\mathbf{C}} \geq 2$ to be satisfied is $K \geq R \geq 2$. This holds for the cases of AF ECGs, where, typically, the number of sources is smaller than the number of leads and greater than 2 .

2) For matrix $\mathbf{F}$ to have full column rank it must fulfill $\sum_{n=1}^{N} I_{n} J_{n} \geq R$. We have that $\sum_{n=1}^{N} I_{n} J_{n} \geq I_{n} J_{n} \geq$ $M_{n}$, for $M_{n} \geq 2$. Since for AF ECGs $M_{n} \gg R>2$, the necessary condition holds. 


\section{EVALUATION ON SYNTHETIC DATA}

The performance of the coupled BTD for ECG source separation is now assessed in two consecutive synthetic AF segments by means of the normalized mean square error (NMSE) between the estimated and original AA signals. The segments contain 3 sources: AA, VA, and a noise source. To simulate the AA signal during AF, the model proposed in [12] that mimics the $\mathrm{f}$ waves is used. This model is given by:

$$
S_{A A}(m)=-\sum_{p=1}^{P} a_{p}(m) \sin (p \theta(m))
$$

with modulated amplitude and phase respectively given by:

$$
a_{p}(m)=\frac{2}{p \pi}\left[a+\Delta a \sin \left(2 \pi \frac{f_{a}}{F_{s}} m\right)\right]
$$

and

$$
\theta(m)=2 \pi \frac{f_{0}}{F_{s}} m+\left(\frac{\Delta f}{F_{f}}\right) \sin \left(2 \pi \frac{F_{f}}{F_{s}} m\right)
$$

where $a$ is the sawtooth amplitude, $\Delta a$ is the modulation peak amplitude, $f_{a}$ is the amplitude modulation frequency, $F_{s}$ is the sampling frequency, $f_{0}$ is the frequency value around which $\theta(m)$ varies sinusoidally, $\Delta f$ is the maximum frequency deviation and $F_{f}$ is the modulation frequency.

In order to simulate the VA signal, a synthetic T-wave modeled by a cosinus function as in [13] and three synthetic QRS complexes modeled by rational functions are generated according to the model function proposed in [14] and added together. This model is given by:

$$
S_{V A}(m)=\mathfrak{R}\left(e^{-i \Theta} r_{a}^{n}\left(e^{i m}\right)\right)
$$

where $\Theta$ is the coefficient parameter and $r_{a}^{n}(\cdot)$ is a basic normalized rational function.

Finally, the noise signal that simulates some interferences present in ECGs is represented by an additive white Gaussian noise (AWGN) with mean and standard deviation given by 0.05 and 0.01 , respectively. The mixing matrix is also Gaussian generated, with scaling factors chosen to obtain an average power ratio between the sources consistent with clinical observations. The synthetic ECG data is generated as the case $\mathrm{A}$ in [12] and divided in two consecutive segments as illustrated in Figure 2.

Coupled BTD is implemented using a data fusion algorithm and BTD is implemented using a non-linear least squares (NLS) method, both available in the software package Tensorlab 3.0 [15], choosing $R=3$ and $L_{r}=17$, for $r=1,2,3$. The choice of $R$ and $L_{r}$ is based on previous experiments, guided by the work of [6]. The tolerance threshold for BTD convergence is set to $10^{-9}$ and the maximum number of iterations is set to 1000 . Ten Monte Carlo runs with Gaussian random initialization for the spatial and temporal factors at each run are used to analyze the AA extraction performance of BTD and coupled BTD in each segment. The best out of the 10 independent initializations is chosen. Monte Carlo runs are needed since the performance of these methods depends strongly on the initialization of their matrix factors and finding a suitable initialization is still an open challenge.

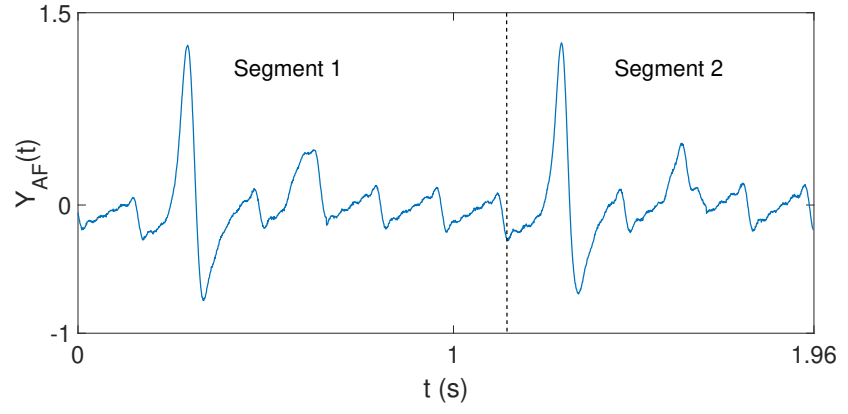

Fig. 2: Generated synthetic signal modeling an ECG with AF patterns plotted in time domain, where $t=m / F_{s}$ are the time values in seconds and $Y_{A F}(t)$ is the amplitude.

TABLE I: NMSE ( $\mathrm{dB}$ ) between the estimated and original AA sources for the coupled BTD and BTD.

\begin{tabular}{|c|c|c|}
\hline Segments & Coupled BTD & BTD \\
\hline \hline $\mathbf{1}$ & -18.9 & -15.4 \\
\hline $\mathbf{2}$ & -19.2 & -13.5 \\
\hline
\end{tabular}

Table I shows the NMSE in $\mathrm{dB}$ between the estimated and original AA sources of segments 1 and 2, for coupled BTD and BTD. It can be seen the AA the sources of both segments are jointly estimated with a satisfactory performance for the coupled BTD, as confirmed by both NMSE values, which are lower than those provided by BTD. In addition, coupled BTD takes 281 iterations to converge to this solution, while BTD takes 283 and 150 iterations to provide a solution for segments 1 and 2, respectively, adding up to a total of 433 iterations. For the record, processing the BTD in the full segment with such configurations does not provide a satisfactory source separation, requiring the choice of higher $L_{r}$ values. Still, the number of iterations to converge for the best solution out of ten Monte Carlo runs is 371.

\section{EVAluATiOn ON REAL DATA}

\section{A. Real Database and Algorithm Setup}

The real recordings belong to a database provided by the Cardiology Department of Princess Grace Hospital Center, Monaco. They are acquired at a $977 \mathrm{~Hz}$ sampling rate and preprocessed as in [7]. Experiments are performed in 5 different segments of ECG recordings from 2 different patients suffering from persistent $\mathrm{AF}$, where 3 of these segments belong to Patient 1, and the other 2 belong to Patient 2. In order to fully exploit the multi-lead diversity, the 12 leads are processed. For a better visualization of the AA, Figure 3 shows lead V1 of the two types of segments used in the reported experiments. The 3 consecutive segments from Patient 1 have 1.20, 0.75 and 0.97 seconds of duration, respectively. The other 2 consecutive segments from Patient 2 have both 1.13 seconds of duration. All the 5 segments are downsampled by a factor of 4 in order to reduce tensor dimensions and subsequent computational time. Previous experiments have shown that downsampling by 


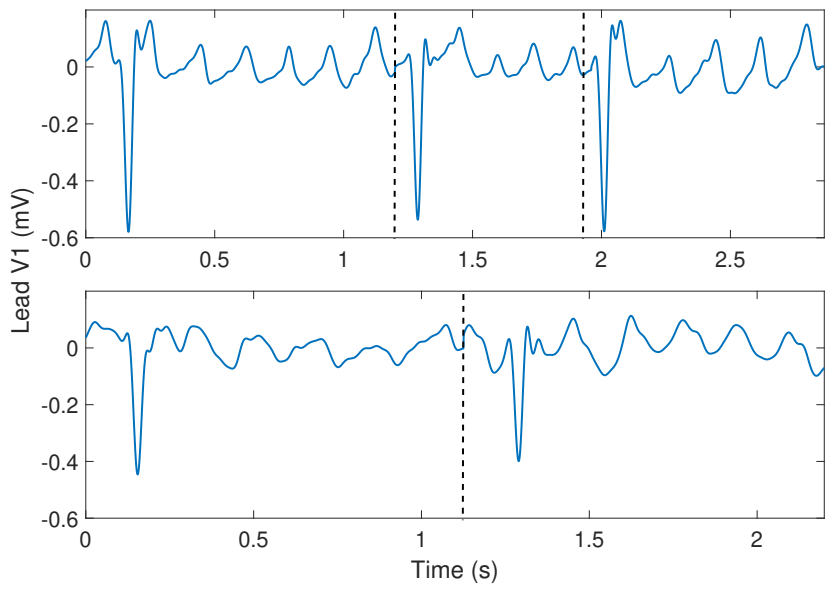

Fig. 3: Top: the AF ECG recording of Patient 1 divided in three consecutive segments of different lengths. Bottom: the AF ECG recording of Patient 2 divided in two consecutive segments of same length. Only lead V1 is shown to ease visualization, although the 12 leads are processed in the experiments reported here.

a factor lower than 10 does not incur any important loss of information in the AF signals of the present database.

Both tensor-based techniques are implemented with the same configuration as in the experiments with synthetic data reported in Section III, changing just the value of the number of sources to $R=4$.

\section{B. AA Content Measurement}

The measurement of the estimation quality (or the AA content) of real signals is a difficult task. Since there is no ground truth for comparison, one needs to take advantage of some features present in AA during AF episodes. For example, in the frequency domain, the AA during $\mathrm{AF}$ has a peak between 3 and $9 \mathrm{~Hz}$. The position of this peak is called dominant frequency (DF). In the present experiments, three parameters are used to measure AA extraction quality. The first one is the spectral concentration (SC), defined as the relative amount of energy around the DF [5]. The next two parameters are the kurtosis, denoted $\kappa$, of the signal in the frequency domain acquired by a 4096-point FFT, and the power contribution to lead $\mathrm{V} 1$, denoted $P(r)$, both proposed in [7] as AA extraction quality indices.

\section{Experimental Results}

Table II shows the values of SC in $\%, \kappa$, and $P(r)$ in $\mathrm{mV}^{2}$ of the estimated AA signal by BTD and coupled BTD, for all the consecutive segments of both patients. The best performance out of the ten independent runs regarding AA estimation quality and source separation is shown.

We can see in Table II that for all the segments of both patients, coupled BTD provides a SC higher than BTD. For the parameters $\kappa$ and $P(r)$, coupled BTD provides higher values
TABLE II: Values of SC (\%), $\kappa$, and $P(r)$ in $\mathrm{mV}^{2}$ of the AA signal estimated by coupled BTD and BTD, for all the processed segments of both patients. The best solution out of ten Monte Carlo runs is chosen.

\begin{tabular}{|c|c|c|c|c|c|}
\hline \multirow{2}{*}{ Coupled BTD } & \multicolumn{3}{|c|}{ Patient 1 } & \multicolumn{2}{c|}{ Patient 2 } \\
\cline { 2 - 6 } & $\mathbf{1}$ & $\mathbf{2}$ & $\mathbf{3}$ & $\mathbf{1}$ & $\mathbf{2}$ \\
\hline \hline SC (\%) & 58.6 & 67.1 & 64.8 & 72.3 & 64.3 \\
\hline$\kappa$ & 138.8 & 94.3 & 143.8 & 138.6 & 207.8 \\
\hline P(r) $\left(\times 10^{-3}\right)$ & 2.3 & 1.2 & 3.3 & 0.9 & 2.0 \\
\hline \hline \multirow{2}{*}{ BTD } & \multicolumn{3}{|c|}{ Patient 1 } & \multicolumn{2}{c|}{ Patient 2 } \\
\cline { 2 - 6 } & $\mathbf{1}$ & $\mathbf{2}$ & $\mathbf{3}$ & $\mathbf{1}$ & $\mathbf{2}$ \\
\hline \hline SC (\%) & 57.4 & 61.8 & 56.3 & 71.2 & 62.7 \\
\hline$\kappa$ & 94.4 & 95.4 & 98.4 & 155.7 & 196.7 \\
\hline P(r) $\left(\times 10^{-3}\right)$ & 0.8 & 1.0 & 3.5 & 0.8 & 2.8 \\
\hline
\end{tabular}

than BTD in all but two segments. From this table one can conclude that both BTD and coupled BTD provide satisfactory AA sources estimates, with a significant superiority of the coupled approach. However, the main advantage of the coupled approach is its ability to jointly estimate more than one segment with the same number of iterations as BTD takes to converge for a single segment, as it will be detailed later in this section.

In addition, when applying BTD to the whole recording, i.e., without dividing it into consecutive segments, the method cannot separate the sources with a low choice of $L_{r}$ and the number of iterations until convergence easily exceeds 1000 . This highlights even more the advantages of segmenting the recording, as the tensor approach can successfully extract the AA from short ECG recordings, whereas techniques based on QRST cancellation, like average beat subtraction and adaptive singular value cancellation, cannot.

Figure 4 illustrates the AA source estimates in the time and frequency domain for Patient 2. At the top it can be seen how the VA is well suppressed, as at the bottom the peak in a frequency between 3 and $9 \mathrm{~Hz}$ is clearly visible, typical of an AA source. It is valid to state that the DF of the AA sources estimated by coupled BTD are exactly the same of the ones estimated by BTD, for all segments of both patients. This shows the consistency of the coupled approach, since BTD has already been validated as an AA extraction tool [16].

Figure 5 compares the number of iterations to provide the best solution by both tensor-based techniques. Unlike coupled BTD, conventional BTD does not jointly estimate the source signals for all segments, so the sum of the number of iterations of each segment is added to each other and shown in the graphs, which provides a number considerably higher compared to the coupled approach. It is also observed that coupled BTD is less sensitive to initialization, as out of the 10 Monte Carlo runs, around $70 \%$ of solutions provided satisfactory source separation performance, whereas only around $50 \%$ of BTD solutions achieved such goal. 

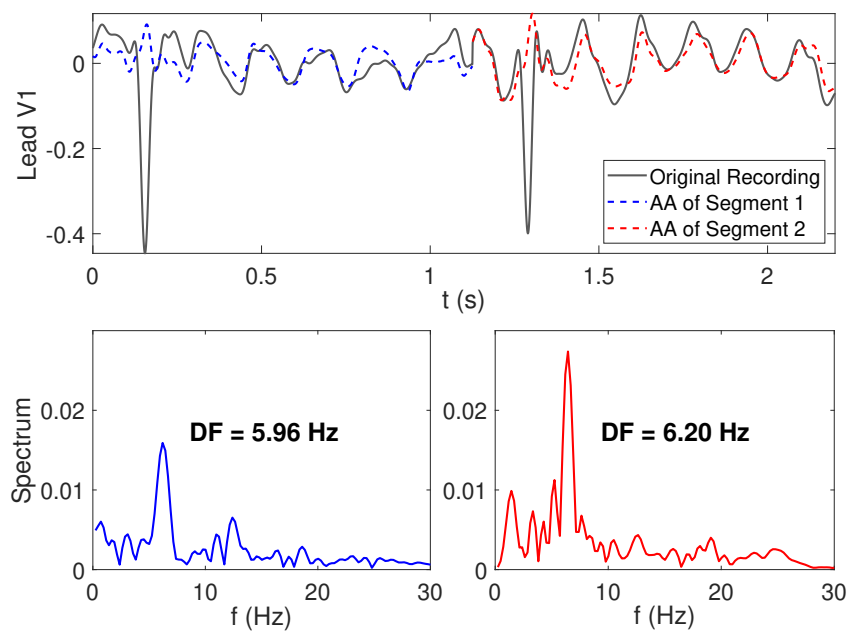

Fig. 4: Estimated atrial sources in lead V1 of Patient 2 by coupled BTD. Top: time domain $(\mathrm{mV})$. Bottom: frequency domain $(\mathrm{mV} / \sqrt{\mathrm{Hz}})$.

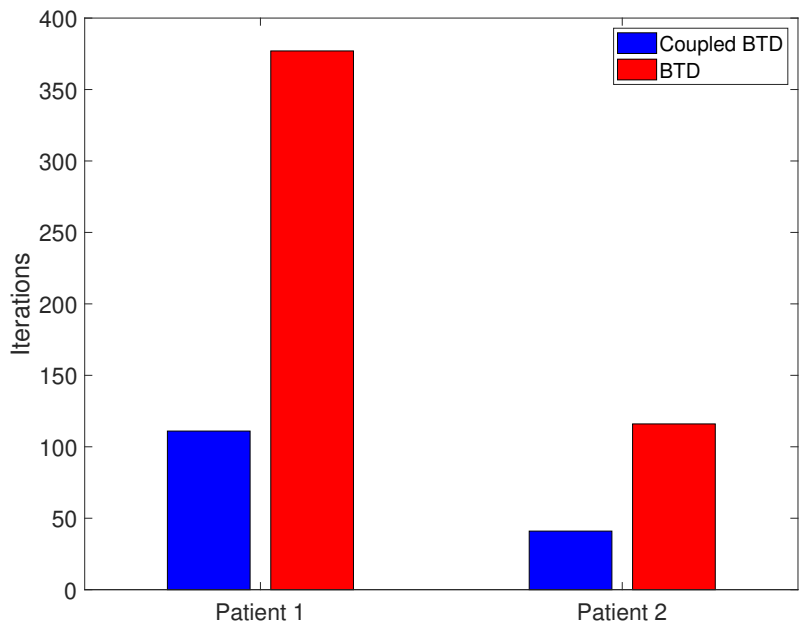

Fig. 5: Total number of iterations until convergence for both tensor-based methods.

\section{CONCLUSION}

Aiming to explore the spatial and temporal diversity of ECG recordings, the present work puts forward a novel noninvasive technique to perform AA extraction from consecutive segments of AF ECGs. The coupled tensor approach is applied for the first time in AF ECG segments, which are modeled as a coupled BTD based on Hankel matrices. Experiments based on Monte Carlo simulations with synthetic and real AF data validate the coupled tensor approach and show its improved performance over the conventional BTD. The coupled approach provides an enhanced source separation compared to the non-coupled version, in terms of AA extraction quality.
However, the main advantage of coupled BTD lies in the fact that it jointly estimates the consecutive segments taking advantage of the temporal diversity of AF ECGs, needing less iterations to converge to a solution and being more robust to initialization.

Future work will focus on building a coupled BTD with alternative signal representations, in order to deal with more complex cases of AF. Assessing coupled BTD with an increased number of segments, using a larger ECG database, is also necessary to confirm its clinical relevance.

\section{REFERENCES}

[1] C. T. January, L. S. Wann, J. S. Alpert, H. Calkins, et al., "2014 AHA/ACC/HRS guideline for the management of patients with atrial fibrillation: A report of the American College of Cardiology/American Heart Association Task Force on practice guidelines and the Heart Rhythm Society", Circulation, vol. 64, no. 21, pp. 2246-2280, 2014.

[2] C. A. Morillo, A. Banerjee, P. Perel, D. Wood, and X. Jouven, "Atrial fibrillation: the current epidemic", Journal of Geriatric Cardiology, vol. 14 , pp. 195-203, 2017

[3] L. Mainardi, L. Sörnmo, S. Cerutti, "Understanding Atrial Fibrillation: The Signal Processing Contribution", Synthesis Lectures on Biomedical Engineering, Morgan \& Claypool Publishers, 2008.

[4] J. J. Rieta, F. Castells, C. Sánchez, V. Zarzoso, and J. Millet, "Atrial activity extraction for atrial fibrillation analysis using blind source separation”, IEEE Transactions on Biomedical Engineering, vol. 51, no. 7, pp. 1176-1186, Jul. 2004.

[5] F. Castells, J. J. Rieta, J. Millet, and V. Zarzoso, "Spatiotemporal blind source separation approach to atrial activity estimation in atrial tachyarrhythmias", IEEE Transactions on Biomedical Engineering, vol. 52, no. 2, pp. 258-267, Feb. 2005.

[6] V. Zarzoso, "Parameter estimation in block term decomposition for noninvasive atrial fibrillation analysis", in Proc. CAMSAP-2017, IEEE International Workshop on Computational Advances in Multi-Sensor Adaptive Processing, Curaçao, Dutch Antilles, Dec. 10-13, 2017.

[7] P. M. R. de Oliveira and V. Zarzoso, "Source analysis and selection using block term decomposition in atrial fibrillation", in Proc. LVA/ICA-2018, 14th International Conference on Latent Variable Analysis and Signal Separation, Guildford, United Kingdom, Jul. 2-6, pp. 46-56, 2018.

[8] L. De Lathauwer, "Blind separation of exponential polynomials and the decomposition of a tensor in rank- $\left(l_{r}, l_{r}, 1\right)$ terms", SIAM Journal on Matrix Analysis and Applications, vol. 32, no. 4, pp. 1451-1474, 2011

[9] B. Hunyadi, W. Van Paesschen, M. De Vos and S. Van Huffel, "Fusion of electroencephalography and functional magnetic resonance imaging to explore epileptic network activity", in Proc. EUSIPCO-2016, 24th European Signal Processing Conference, Budapest, Hungary, Aug. 28 Sep. 2, pp. 240-244, 2016.

[10] E. Acar, Y. Levin-Schwartz, V. D. Calhoun and T. Adali, "ACMTF for fusion of multi-modal neuroimaging data and identification of biomarkers", in Proc. EUSIPCO-2017, 25th European Signal Processing Conference, Kos, Greece, Aug. 28 - Sep. 2, pp. 643-647, 2017.

[11] M. Sørensen and L. de Lathauwer, "Coupled canonical polyadic decompositions and (coupled) decompositions in multilinear rank$\left(L_{r, n}, L_{r, n}, 1\right)$ terms - part I: uniqueness", SIAM J. Matrix Anal. Appl., vol. 36, pp. 496-522, 2015.

[12] M. Stridh and L. Sornmo, "Spatiotemporal QRST cancellation techniques for analysis of atrial fibrillation", IEEE Transactions on Biomedical Engineering, vol. 48, no. 1, pp. 105-111, 2001.

[13] P. Dolinský, I. Andráš, L. Michaeli and D. Grimaldi, "Model for generating simple Synthetic ECG signals", Acta Electrotechnica et Informatica, vol. 18, no. 3, pp. 3-8, 2018.

[14] G. Bognár and F. Schipp, "Geometric interpretation of QRS complexes in ECG signals by rational functions", Annales Univ. Sci. Budapest., Sect. Comp., vol. 47, pp. 155-166, 2018.

[15] N. Vervliet, O. Debals, L. Sorber, M. Van Barel, and L. De Lathauwer, Tensorlab 3.0, Available online, Mar. 2016. URL: https://www.tensorlab.net/

[16] P. M. R. de Oliveira and V. Zarzoso, "Block term decomposition of ECG recordings for atrial fibrillation analysis: Temporal and inter-patient variability", Journal of Communication and Information Systems, vol. 34, no. 1, pp. 111-119, 2019. 